\title{
Plasma Lipoprotein-associated Phospholipase A2 and Superoxide Dismutase are Independent Predicators of Cognitive Impairment in Cerebral Small Vessel Disease Patients: Diagnosis and Assessment
}

\begin{abstract}
Shuzhen Zhu', ${ }^{1, \#}$ Xiaobo Wei ${ }^{1, \#, ~ X i a o h u a ~ Y a n g, " \#, ~ Z i f e n g ~ H u a n g 1, \#, ~ Z i h a n ~ C h a n g 1, ~ F e n ~ X i e ~}{ }^{1}$, Qin Yang $^{1}$, Changhai Ding ${ }^{2,3}$, Wei Xiang ${ }^{4}$, Hongjun Yang ${ }^{4}$, Ying Xia ${ }^{5}$, Zhong-Ping Feng ${ }^{6}$, Hong-Shuo Sun $^{6,7}$, Midori A. Yenari ${ }^{8}$, Lin Shi ${ }^{9}, 10$, Vincent CT Mok ${ }^{11, *}$, Qing Wang,**

${ }^{1}$ Department of Neurology and ${ }^{2}$ Department of Orthopedics, Zhujiang Hospital of Southern Medical University, Guangdong, China. ${ }^{3}$ Menzies Institute for Medical Research, University of Tasmania, Hobart, Australia. ${ }^{4}$ Department of Neurology, Guangzhou General Hospital of Guangzhou Military Command, Guangdong, China. ${ }^{5}$ Shanghai Key Laboratory of Acupuncture Mechanism and Acupoint Function, Fudan University, Shanghai, China. ${ }^{6}$ Department of Physiology and ${ }^{7}$ Department of Surgery, Faculty of Medicine, University of Toronto, Toronto, Ontario M5S 1A8, Canada. ${ }^{8}$ Department of Neurology, University of California, San Francisco \& the San Francisco Veterans Affairs Medical Center, San Francisco, USA. ${ }^{9}$ Department of Imaging and Interventional Radiology, The Chinese University of Hong Kong, Hong Kong, China. ${ }^{10}$ BrainNow Research Institute, Shenzhen, China. ${ }^{11}$ Gerald Choa Neuroscience Centre, Department of Medicine and Therapeutics, Faculty of Medicine, Prince of Wales Hospital, The Chinese University of Hong Kong, Hong Kong, China.
\end{abstract}

[Received January 6, 2018; Revised March 1, 2019; Accepted March 4, 2019]

\begin{abstract}
Lipoprotein-associated phospholipase A2 (Lp-PLA2) and superoxide dismutase (SOD) are linked to regulating vascular/neuro-inflammation and stroke. Using a retrospective design, we investigated whether circulating Lp-PLA2 and SOD in cerebral small vessel disease (CSVD) patients were associated with cognitive impairment. Eighty-seven CSVD patients were recruited. Plasma Lp-PLA2 and SOD were determined, and cognitive status was measured by the Mini-Mental State Examination (MMSE) and Montreal Cognitive Assessment (MoCA). The severity of white matter hypoerintensities (WMHs) in CSVD patients was rated according to Fazekas scales, and LpPLA2/SOD levels and MMSE/MoCA were compared. Multiple linear regressions were used to evaluate the relationship between Lp-PLA2 and SOD and the cognitive impairment. Ordinal logistic regression and generalized linear models (OLRGLMs) were applied to confirm whether Lp-PLA2 and SOD are independent risk factors for cognitive impairment in CVSD. Lp-PLA2 and SOD with mild or severe cognitive impairment were lower than those with normal congnition. Lp-PLA2 and SOD in CSVD patients with severe WMHs were significantly lower than those with mild or moderate WMH lesions. We noted positive linear associations of Lp-PLA and SOD with cognitive impairment in CSVD, independent of LDL-C. OLRGLMs confirmed that Lp-PLA2 and SOD were independent risk factors of cognitive impairment in CSVD. Lp-PLA2 and SOD are independently associated with cognitive impairment and WMH lesion, and may be useful for the rapid evaluation of cognitive impairment in CSVD. Lp-PLA2/SOD are modifiable factors that may be considered as therapeutic targets for preventing cognitive impairment in CSVD.
\end{abstract}

Key words: Lp-PLA2, superoxide dismutase, cerebral small vessel disease, cognition

\footnotetext{
*Correspondence should be addressed to: Dr. Qing Wang, Zhujiang Hospital, Southern Medical University, Guangzhou, China. Email: denniswq@yahoo.com; Prof. Vincent CT Mok, Faculty of Medicine, Prince of Wales Hospital, The Chinese University of Hong Kong, Hong Kong, China. Email: vctmok@,cuhk.edu.hk. "These authors contributed equally.
}

Copyright: () 2019 Zhu S et al. This is an open-access article distributed under the terms of the Creative Commons Attribution License, which permits unrestricted use, distribution, and reproduction in any medium, provided the original author and source are credited. 
Cerebral small vessel disease (CSVD) is a highly prevalent condition associated with neuroinflammation and cognitive impairment $[1,2]$. However, the diagnosis and classification of CSVD with cognitive impairment were somewhat difficult for community doctors or nonStroke subspecialist. Clinically, there is no blood marker available to diagnose and assess CSVD with cognitive impairment in clinical settings [3,4]. Worldwide, the number of CSVD patients is more common in Asians than in Caucasians [5]. Growing evidence has shown that systemic inflammatory mediators such as superoxide dismutase (SOD) and lipoprotein-associated phospholipase A2 (Lp-PLA2) are associated with CSVD and cognitive impairment [4, 6-8]. However, the role of LpPLA2 and SOD, especially SOD, in CSVD patients with cognitive impairment is largely unknown.

Lp-PLA2 acts as an enzyme and proinflammatory marker of cardio- and cerebrovascular risk; however, some studies have indicated that Lp-PLA2 exerts antioxidative and anti-inflammatory functions under certain circumstances [9]. Lp-PLA2 circulates in plasma in its active form as a complex with low-density lipoprotein (LDL) and high-density lipoprotein (HDL). SOD is an enzyme that catalyzes the dismutation of the superoxide radical into $\mathrm{H}_{2} \mathrm{O}_{2}$ and oxygen and has powerful anti-inflammatory activity $[10,11]$. A recent study in humans suggested that plasma SOD could be a marker of vascular alterations. Other studies have also indicated that higher levels of Lp-PLA2 are associated with cerebral ischemic stroke and cognitive impairment [7, $8,11]$; however, the roles of SOD in cognitive impairment remain unexplored.

To explore the association of Lp-PLA2 and SOD with CSVD patients with cognitive impairment, we asked whether (1) plasma Lp-PLA2 and SOD levels in CSVD patients with cognitive impairment were higher or lower than those in CSVD patients with normal cognition [NC], (2) there was a certain linear relationship between LpPLA2/SOD and cognitive impairment in CSVD, and (3) the Lp-PLA2/SOD-related receiver operating characteristic (ROC) curve could be used to distinguish CSVD with mild or severe cognitive impairment [MCI, $\mathrm{SCI}$ ] from those with NC.

\section{MATERIALS AND METHODS}

\section{Study design}

This study was a retrospective analysis of collected data from patients admitted with subcortical white matter hyperintensity (WMH) and lacunar infarct in two medical centers (Zhujiang hospital and Guangzhou General Hospital of Military Command, Guangzhou, China). The study was approved by the ethics Human Research
Committee at Zhujiang Hospital of Southern Medical University and complied with the principles outlined in the revised Declaration of Helsinki of 1975 and the National Institutes of Health Human Subjects Policies and Guidelines released in 1999. Informed written or verbal consent was obtained from patients and family members.

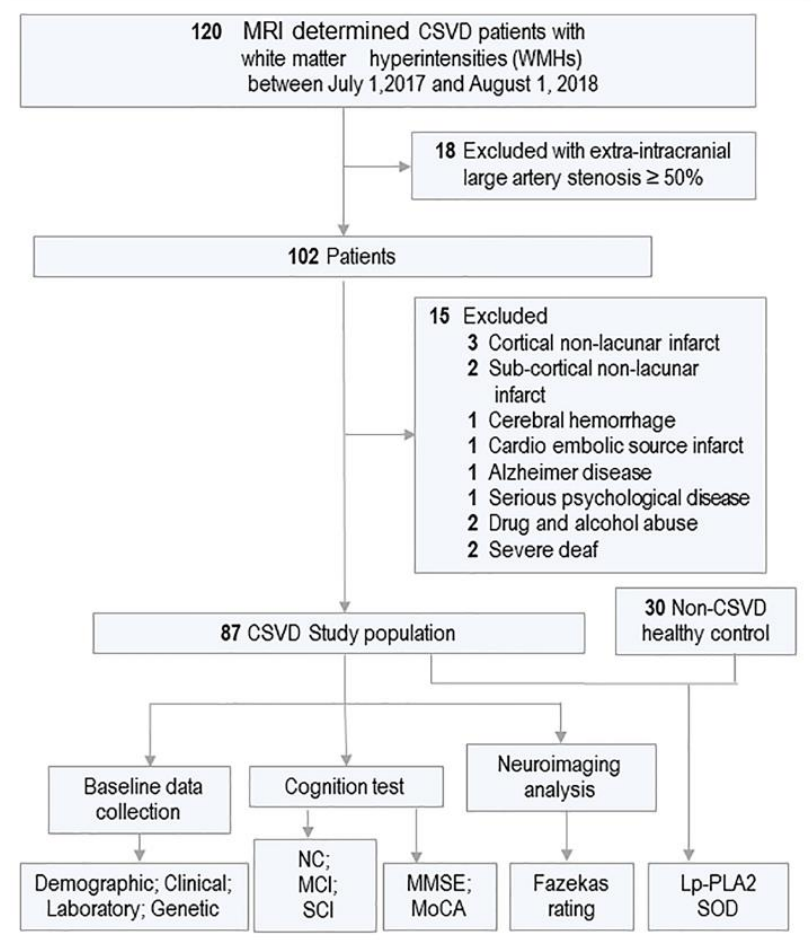

Figure 1. All 120 CSVD patients who met the clinical diagnostic criteria of white matter hyperintensities were enrolled in this study from Jul 2017 to Aug 2018. Of 33 participants met the excluded criteria and 87 participants were included in this study for further analyzing. 30 age and gendermatched non-CSVD healthy controls (HC) were enrolled from phy sical examnination center. The plasma level of Lp-PLA2 and SOD was compared between CSVD and HC groups. In addition, The cognitive function and white matter hyperintensity were evaluated and the variables were collected and analyzed among groups.

\section{Patient selection}

One-hundred and twenty consecutive CSVD participants with Magnetic Resonance Imaging (MRI)-determined subtypes (WMH and lacunar infarcts) were recruited from July 2017 to August 2018. All participants were clinically diagnosed with CSVD at Zhujiang hospital and Guangzhou General Hospital of Military Command based on the criteria of Trial of Org 10172 in Acute Stroke Treatment [12]. All participants underwent T2, T1 turbo field echo, fluid-attenuated inversion recovery (FLAIR) 
imaging using a 3.0-T MRI scanner (Philips 3.0T Achieva; Philips Healthcare, Andover, MA). Subcortical WMHs were defined ac cording to the study of Mok [13]. A lacune was defined as a round or ovoid lesion $\geq 3 \mathrm{~mm}$ and $\leq 15$ $\mathrm{mm}$ in diameter found on T1-weighted and T2-weighted images with a perilesional halo on FLAIR images as defined by Wardlaw et al [14]. The study flow diagram was shown in Figure 1. Of the 120 participants, we excluded 18 patients who showed extra-intracranial large artery stenosis $\geq 50 \%$ and 3 who exhibited a cortical nonlacunar infarct. Others with subcortical nonlacunar infarct $(n=2)$, cerebral hemorrhage $(n=1)$, cardio embolic source infarct $(n=1)$, Alzheimer disease $(A D ; n=2)$, serious psychological disease $(n=2)$, drug and alcohol abuse $(n=2)$, severe deaf $(n=2)$, were also excluded from this study. Thus, the final analysis was performed in 87 CSVD eligible participants. In addition, 30 age-and gender-matched healthy control (HC) subjects were recruited at the physical examination center and the plasma levels of Lp-PLA2 and SOD were collected. We received written informed patient consent to perform this study.

\section{Participant characteristics}

Eligible participant characteristics that were regarded as likely relevant confounders of cognition or Lp-PLA2 were recorded as baseline data. In this study, the following variables were collected: 1) demographic data (gender, age, years of education); 2) clinical data (body mass index [BMI], history of hypertension, diabetes and coronary heart disease [CHD], medical history of statin and antithrombotic use), with hypertension and diabetes mellitus defined as a self-reported medical diagnosis, antihypertensive/antidiabetic medication use, or a new diagnosis according to increased fasting and postprandial blood glucose, or CHD defined as a self-reported medical diagnosis or heart disease medication use, and BMI calculated as weight divided by height squared $\left.\left(\mathrm{kg} / \mathrm{m}^{2}\right) ; 3\right)$ laboratory markers such as cholesterol metabolismrelevant biomarkers including LDL-cholesterol (LDL-C), HDL-cholesterol (HDL-C) and total cholesterol, kidney function-relevant biomarkers including urea, creatinine $(\mathrm{Cr})$ and UA, and ROS markers including Lp-PLA2 and SOD; 4) genetic data such as apolipoprotein E (APOE) allele carrier status.

\section{Cognitive impairment severity grading}

The diagnosis of cognitive impairment was performed by two neruologists according to the criteria proposed by Philip B., which was recommeded by Guidelines for Cerebral Vascular Diseases[15]. Cognitive domains including executive/attention, memory, language, and visuospatial functions were assessed. A decline in cognitive function from a prior baseline in at least one cognitive domain and normal or mildly impaired instrumental activities of daily living were defined as MCI. A deficit in performance in $\geq 2$ cognitive domains that are of sufficient severity to affect the subject's activities of daily living was defined as SCI. The Mini-Mental State Examination (MMSE) and Montreal Cognitive Assessment (MoCA) were evaluated on the day of administration.

\section{Laboratory biomarker measurem ents}

Total cholesterol, HDL-C, LDL-C, urea, leukocyte, neutrophile granulocyte (NEU), hypersensitive C-reactive protein (hs-CRP), erythrocyte sedimentation rate (ESR), $\mathrm{Cr}$, UA, Lp-PLA2, APOE and SOD in the plasma were measured twice during hospitalization. Fasting blood samples were collected in tubes containing EDTA and centrifuged at $3000 \mathrm{~g}$ for $10 \mathrm{~min}$, and aliquots of plasma were stored at $-70^{\circ} \mathrm{C}$ until use for biochemical analyses. Since the Lp-PLA2 mass test only detects a portion of the total Lp-PLA2, mainly the Lp-PLA2 associated with HDL, which is the "good cholesterol" that displays protective effects [16], and we wanted to explore the neuroprotective effects of plasma Lp-PLA2, therefore here we only measured Lp-PLA2 mass in the current study. Lp-PLA2 mass was detected with the Human PLA2G7/PAF-AH/Lp-PLA2 Quantikine Enzyme-linked Immunosorbent Assay (ELISA) Kit (Bio-Techne Corporation, $R \& D$ system, Minnesota) according to the referenced instructions by Packard et al. and the manufacturer's instructions [17]. Briefly, $10 \mu \mathrm{L}$ EDTA plasma was added to a 96-well plate precoated with an antibody specific to Lp-PLA2. After incubation with the Lp-PLA2-specific primary and biotin-conjugated antibodies, the plasma concentration of Lp-PLA2 was determined by comparing the optical density (O.D.) of the samples to the standard curve. Plasma SOD levels were measured with a $\mathrm{Cu} / \mathrm{Zn}$ SOD ELISA kit (Bio-Techne Corporation, R\&D system, Minnesota, USA) ac cording to the manufacturer's instructions. Absorbance was read on a spectrophotometer (Thermo Luminoskan Ascent, Waltham MA, USA) at $450 \mathrm{~nm}$ [10].

APOE genotyping was detected by Q-PCR as described by Christensen et al., [18]. Genomic DNA was extracted from blood samples using Qiagen DNA Blood kits (\#51162; Qiagen Inc., Valencia, CA, USA). SNPspecific primers and probes were designed by Invitrogen (Invitrogen,). TaqMan real-time PCR assays were performed in an ABI $7900 \mathrm{HT}$ machine, using the following cycling program: $95^{\circ} \mathrm{C}$ for $10 \mathrm{~min}$ followed by 40 cycles of $95^{\circ} \mathrm{C}$ for $15 \mathrm{sec}$ and $60^{\circ} \mathrm{C}$ for $1 \mathrm{~min}$. 
A

Lp-PLA2 and SOD between groups (HC VS. CSVD)

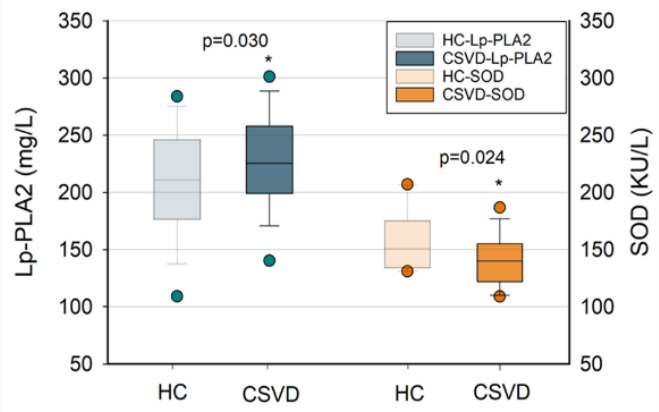

C

Linear regression of Lp-PLA2 and SOD (CSVD)

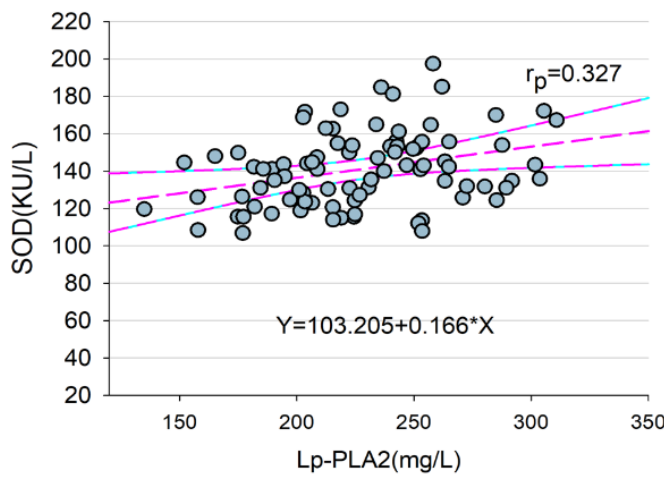

B

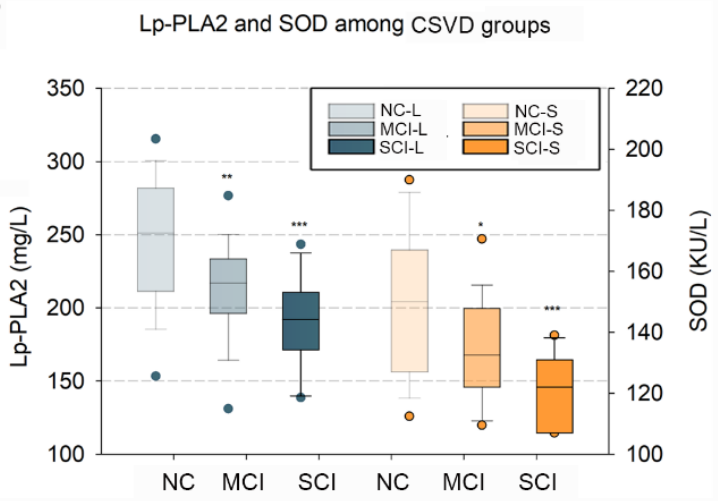

Figure 2. Comparison of biomarkers for Lp-PLA2 and SOD levels in the CS VD patients with different severity of cognitive impairment. (A) Comparison was made using independent $t$-test between the CSVD patients and $\mathrm{HC}$ (healthy control). The level of Lp-PLA2 increased and SOD decreased in CSVD patients. (B) Comparisons were made using one-way covariance with adjusted for the confounders (sex, age, education, BMI, cholesterol, HDL-C, LDL-C, urea, Cr, UA, hy pertension, diabetes, CHD, APOE genoty pe, and medication use) followed by a post hoc Bonferroni test. Cognitive impairment group (MCI and SCI) displayed significantly lower levels of Lp PLA2 and SOD than the normal cognition (NC) group (see also table 1). (C) The plasma Lp-PLA2 was linear correlated with SOD ( $r=0.327, \mathrm{p}<0.05)$. Abbreviations: APOE, apolipoprotein E; BMI, body mass index; CHD, coronary heart disease; Cr, creatinine; CSVD, cerebral small vessel disease; HC, healthy control; HDL-C, Highdensity lipoprotein cholesterol; LDL-C, low-density lipoprotein cholesterol; Lp-PLA2, Lipoprotein-associated Phospholipase A2; MCI: mild cognitive impairment; MMSE, Mini-Mental State Examination; MoCA, Montreal Cognitive Assessment; NC, normal cognition; SCI, severe cognitive impairment; SOD, Superoxide Dismutase; UA, uric acid. * Compared to $\mathrm{NC} p<0.05$, ** Compared to NC $p<0.01$, *** Compared to NC $p<0.001$, ${ }^{\# \#}$ Compared to $\mathrm{MCI} p<0.01$.

\section{The rating of white matter hyperintensities with Fazekas scales}

MRI including T2 and FLAIR sequences were used in this study. The severity of white matter hypoerintensities (WMHs) was rated according to Fazekas scales by two raters. [19] Acc ording to the scores of Fazekas, the CSVD patients were divided into three groups (1: Mild WMHs, 2: Modest WMHs, 3, Severe WMHs).

\section{Statistical Analysis}

All data analyses were conducted using SPSS 20.0 (IBM
Corporation, Armonk, NY, USA). A * $p$-value $<0.05$ was considered significant. All continuous variables, for example age, BMI, MoCA score, MMSE score, and LpPLA2, SOD, UA and lipid concentrations, are shown as the mean $\pm \mathrm{SD}$; all categorical variables, such as gender, APOE genotype, hypertension, and diabetes, are presented as a percentage. Signific ant differences in LpPLA2 and SOD between healthy and CSVD subjects were analyzed with independent $t$-test; while differences in plasma Lp-PLA2 and SOD levels among CSVD patients with different degrees of cognitive impairment, and differences in Lp-PLA2 and SOD levels among CSVD patients with different degrees of WMHs were analyzed 
with one-way covariance followed by a post hoc Bonferroni test. Pearson correlation analysis was used to examine the relationship between Lp-PLA2 and other risk factors. Multiple linear regressions with a backward elimination model were used to detect the adjusted partial linear correlation between Lp-PLA2/SOD and MMSE/MoCA scores. Ordinal logistic regression and generalized linear models (OLRGLMs) were used to detect the significantly independent associated factors for cognitive decline in CSVD. To assess the diagnostic value of Lp-PLA2 and SOD in the identification of CSVD with MCI or SCI and discrimination from CSVD with NC, ROC analysis was conducted, and the ROC curve for the combination of Lp-PLA2 and SOD was calculated to screen for a better diagnosis tool.

Table 1. Baseline Characteristic of Study CSVD Population.

\begin{tabular}{|c|c|c|c|c|c|}
\hline \multirow{2}{*}{ Variable } & \multicolumn{5}{|c|}{ Subgroup comparison } \\
\hline & $\mathrm{NC}(\mathrm{N}=46)$ & $\operatorname{MCI}(\mathbf{N}=30)$ & $\operatorname{SCI}(N=11)$ & F or $X^{2} *$ & p value \\
\hline \multicolumn{6}{|l|}{ Demographic } \\
\hline Age, mean (SD), y & $63.9(10.1)$ & $67.9(12.1)$ & $74.2(13.7)$ & 4.039 & $.021^{\#}$ \\
\hline Male, N (\%) & $29(63.4)$ & $19(63.3)$ & $8(72.7)$ & 0.384 & .902 \\
\hline Education. mean (SD). v & $9.0(3.4)$ & $7.5(2.1)$ & $6.6(2.7)$ & 4.243 & $n 1 e^{\# \#}$ \\
\hline \multicolumn{6}{|l|}{ Clinical } \\
\hline BMI, mean (SD) & $23.9(2.8)$ & $23.1(3.2)$ & $24.0(4.5)$ & 0.600 & .551 \\
\hline Hypertension, N (\%) & $28(60.9)$ & $16(53.3)$ & $9(81.80)$ & 2.743 & .274 \\
\hline Diabetes, N (\%) & $13(28.3)$ & $7(23.3)$ & $4(36.4)$ & 0.706 & .706 \\
\hline CHD, N (\%) & $20(43.5)$ & $11(36.7)$ & $7(63.6)$ & 2.381 & .301 \\
\hline Statin Use, N (\%) & $43(93.5)$ & $24(80.0)$ & $9(81.8)$ & 3.336 & .165 \\
\hline Antithrombotic use, N (\%) & $44(95.7)$ & $24(80.0)$ & $9(81.8)$ & 4.926 & 0.072 \\
\hline \multicolumn{6}{|l|}{ Laboratory mean(SD) } \\
\hline Cholesterol, (mmol/L) & $4.8 \pm 1.0$ & $4.4 \pm 1.0$ & $4.1 \pm 1.1$ & 2.874 & .062 \\
\hline HDL-C, $(\mathrm{mmol} / \mathrm{L})$ & $1.3 \pm 0.5$ & $1.2 \pm 0.4$ & $1.2 \pm 0.2$ & 0.621 & .540 \\
\hline LDL-C, $(\mathrm{mmol} / \mathrm{L})$ & $2.7 \pm 0.9$ & $2.6 \pm 0.7$ & $2.5 \pm 0.9$ & 0.561 & .573 \\
\hline Urea. $(\mathrm{mmol} / \mathrm{L})$ & $5.3 \pm 1.4$ & $6.0 \pm 1.5$ & $6.0 \pm 2.9$ & 1.909 & .155 \\
\hline $\mathrm{Cr},(\mathrm{umol} / \mathrm{L})$ & $78.6 \pm 28.1$ & $84.2 \pm 34.1$ & $97.7 \pm 36.5$ & 1.690 & .191 \\
\hline UA, (umol/L) & $408.8 \pm 115.0$ & $384.3 \pm 110.2$ & $386.3 \pm 122.2$ & 0.478 & .622 \\
\hline Leukocyte, $(\mathrm{G} / \mathrm{L})$ & $6.8 \pm 1.8$ & $7.6 \pm 2.4$ & $7.0 \pm 1.5$ & 1.456 & 0.239 \\
\hline NEU, (G/L) & $4.2 \pm 1.4$ & $4.9 \pm 2.2$ & $4.8 \pm 1.4$ & 1.702 & 0.189 \\
\hline NEU & $60.8 \pm 9.0$ & $62.7 \pm 9.9$ & $67.3 \pm 7.3$ & 2.256 & 0.111 \\
\hline Hs-CRP, (mg/L) & $3.4 \pm 4.1$ & $4.4 \pm 10.2$ & $3.0 \pm 2.0$ & 0.143 & 0.867 \\
\hline $\mathrm{ESR},(\mathrm{mm} / \mathrm{H})$ & $22.4 \pm 17.0$ & $30.2 \pm 28.7$ & $23.6 \pm 18.3$ & 0.752 & 0.476 \\
\hline Lp-PLA2 mass, (mg/L) & $246.2 \pm 45.4$ & $212.4 \pm 35.3$ & $189.2 \pm 30.7$ & 11.82 & $<0.001 * * *$ \\
\hline SOD, (KU/L) & $149.6 \pm 24.3$ & $135.4 \pm 16.4$ & $120.7 \pm 12.3$ & 10.354 & $<0.001 * * *$ \\
\hline \multicolumn{6}{|l|}{ Genetic } \\
\hline APOE4 carrier, No. $(\%)$ & $6(13.0)$ & $3(10.0)$ & $1(9.1)$ & 0.237 & .897 \\
\hline
\end{tabular}

Data shown are mean \pm S.D. The comparison s of Lp-PLA2 and SOD among the groups were done with one-way covariance with adjusted for the confounders (see also Fig. 3). Comparisons of confounders were made using one-way ANOVA followed by Bonferroni post-hoc tests. Values with $p<0.05$ was regarded as significant related to mult iple comparisons. Abbreviations: NC, normal cognition; MCI: mild cognitive impairment; SCI, severe cognitive impairment; MMSE, Mini-Mental State Examination (range, 0-30, with 30 a perfect score); BMI, body mass index; CHD, coronary heart disease; HDL-C, High-density lipoprotein cholesterol; LDL-C, low-density lipoprotein cholesterol; Cr, creatinine; UA, uric acid; Che, Cholinesterase; APOE, apolipoprotein E. Lp-PLA2, Lipoprotein-associated Phospholipase A2; SOD, Superoxide Dismut ase; NEU, Neutrophile granulocyte; hs-CRP, hypersensitive C-reactive protein; ESR, Erythrocyte sedimentation rate. ${ }^{\#}$ Compared to NC $p=0.023,{ }^{\# \#}$ Compared to NC $p=0.05$ 


\section{RESULTS}

\section{Participant characteristics}

Eighty-seven participants were successfully screened and enrolled in the study, and neuropsychologic al test (MMSE and MoCA) evaluations were acquired for each participant. Forty-six of the patients with CSVD had NC (MMSE score exceeded 24 points), whereas 30 of the CSVD patients were classified as having MCI (MMSE score ranged from 18 to 23) and 11 participants as having SCI with an MMSE score no more than 17 points. Patients characteristics, including sex, age, BMI, past medical history (hypertension, diabetes and heart disease), plasma biomarkers, medication history (antithrombotic, lipidregulating drugs), biomarkers of systemic inflammation (leukocyte, hs-CRP, neutrophile granulocyte, ESR) and APOE genotype, were summarized and included in this study (Table 1). Interestingly, besides significant differences were found among the groups in age $(\mathrm{F}=4.039$, $p=0.021)$ and years of education $(\mathrm{F}=4.243, p=0.018)$, we noted signifcant differences of Lp-LPA2 (F=11.82, $p<0.001)$ and $\mathrm{SOD}(\mathrm{F}=10.354, p<0.001)$ among NC, MCI and SCI groups. However, no signific ant differences in BMI, cholesterol, HDL-C, LDL-C, leukocyte, hs-CRP, neutrophile granulocyte, ESR, Urea, $\mathrm{Cr}$, UA, sex, hypertension, diabetes, CHD, APOE genotype, or medication use among three groups were observed in this study.

\section{Comparison of plasma Lp-PLA2 and SOD levels in CSVD patients}

Plasma Lp-PLA2 and SOD levels in CSVD patients across all groups were higher than those in HC (Fig. 2A). Analysis of covariance showed signific ant differences in Lp-PLA2 and SOD levels among the CSVD groups (SCI, $\mathrm{MCI}$ and $\mathrm{NC}$ ) after adjusting other confounders for sex, age, education, BMI, cholesterol, HDL-C, LDL-C, urea, $\mathrm{Cr}$, UA, hypertension, diabetes, CHD, APOE genotype, and medication use $(\mathrm{F}=11.82, p<0.001$ for Lp-LPA2; $\mathrm{F}=10.354, p<0.001$ for SOD, Table 1). Bonferroni post hoc comparisons showed that there were significant differences in Lp-PLA2 and SOD levels between the NC and MCI ( $p=0.002$ for Lp-PLA2; $p=0.013$ for SOD) and between the NC and SCI groups ( $p<0.001$ for Lp-PLA2; $p p<0.001$ for SOD, Fig. 2B). To evaluate whether there was a relationship between Lp-PLA2 and SOD levels, Pearson's correlation between plasma Lp-PLA2 and SOD levels was analyzed. Lp-PLA2 levels were positively correlated with SOD levels $(\mathrm{r}=0.327, p<0.001)$, showing a linear relationship (Fig. 2C).
Table 2. Ordinal logistic regression of possible variables as sociated with cognitive decline in CSVD.

\begin{tabular}{|c|c|c|}
\hline \multirow{2}{*}{ Variable } & \multicolumn{2}{|c|}{$\mathbf{O R}$} \\
\hline & $\begin{array}{l}\text { Adjusted OR } \\
\quad(95 \% \text { CI) }\end{array}$ & p value \\
\hline Age (years) & $1.050(0.991-1.120)$ & 0.097 \\
\hline BMI & $0.887(0.715-1.100)$ & 0.275 \\
\hline Education (years) & $0.693(0.528-0.908)$ & $0.008 * *$ \\
\hline Hypertension & $0.652(0.154-2.759)$ & 0.561 \\
\hline Diabetes, n (\%) & $2.176(0.450-10.525)$ & 0.334 \\
\hline CHD, n (\%) & $2.236(0.522-9.528)$ & 0.278 \\
\hline Cholesterol (mmol/L) & $0.562(0.265-1.195)$ & 0.134 \\
\hline HDL cholesterol $(\mathrm{mmol} / \mathrm{L})$ & $0.966(0.182-5.109)$ & 0.080 \\
\hline LDL cholesterol $(\mathrm{mmol} / \mathrm{L})$ & $2.601(0.942-7.184)$ & 0.065 \\
\hline Urea $(\mathrm{mmol} / \mathrm{L})$ & $1.521(1.015-2.280)$ & 0.042 \\
\hline $\mathrm{Cr}(\mathrm{umol} / \mathrm{L})$ & $1.002(0.981-1.024)$ & 0.848 \\
\hline $\mathrm{UA}(\mathrm{umol} / \mathrm{L})$ & $1.002(0.996-1.008)$ & 0.466 \\
\hline SOD (KU/L) & $0.933(0.895-0.972)$ & $0.001 * *$ \\
\hline Lp-PLA2 mass (mg/L) & $0.977(0.962-0.992)$ & $0.003 * *$ \\
\hline Antithrombotic use, n (\%) & $8.069(0.327-199.032)$ & 0.202 \\
\hline Statin use, $\mathrm{n}(\%)$ & $0.871(0.048-15.196)$ & 0.925 \\
\hline
\end{tabular}

\section{Comparison of plasma levels of Lp-PLA2 and SOD in CSVD with different severity of WMHs}

Both MMSE $(\mathrm{F}=5.582, p<0.01)$ and MoCA $(\mathrm{F}=3.488$, $p<0.05)$ scores significantly decreased in severe WMHs patients as showed in Figure 3D and E. According to the scores of Fazekas, the CSVD patients were divided into three groups as classified by the severity of WMH (1: Mild WMHs, 2: Modest WMHs, 3, Severe WMHs), which was shown in Figure 3A. The relatively lower levels of Lp-PLA2 $(\mathrm{F}=6.522, p<0.01)$ and SOD $(\mathrm{F}=3.396$, $p<0.05)$ were observed in severe WMHs subjects compared to those in the mild group (Fig. 3B, C).

\section{Linear correlation between plasma Lp-PLA2/SODlevels and MMSE or MoCA scores}

The results from the backward elimination for multiple linear regression with adjustment for LDL-C and other 
parameters including age, gender, degree of education, hypertension, diabetes mellitus, CHD, statin use, antithrombotic use, HDL-C, cholesterol, urea, and $\mathrm{Cr}$ are presented in Supplementary Table 1 and Figure 4. The correlations between Lp-PLA2 and MMSE, and between SOD and MMSE were analyzed (Pearson correlation coefficient: 0.438 and 0.397 , respectively, Fig. 4A, C). Linear regression analysis (Fig. 4A, C) showed that there $w$ as a linear relationship between Lp-PLA2 and MMSE, with linear regression equation $Y=12.182+0.046^{*} \mathrm{X}$, and between SOD and MMSE, with linear regression equation $\mathrm{Y}=11.093+0.081 * \mathrm{X}$. Furthermore, another cognition evaluation tools, MoCA was also applied in this study to confirm the relationship between Lp-PLA2/SOD, and results showed that a linear relationship was also found between Lp-PLA2/SOD and MoCA scores. The linear regression equation was $\mathrm{Y}=6.780+0.049 * \mathrm{X}$ between $\mathrm{Lp}$ PLA2 and MoCA, and $Y=2.103+0.111 * X$ between SOD and MoCA scores (Fig. 4B, D).

A

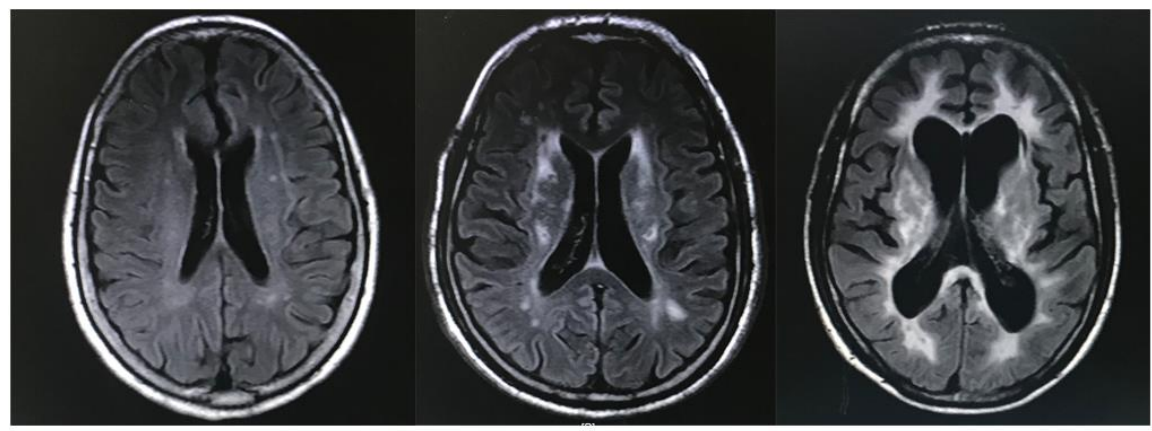

Fazekas 1

Fazekas 2

Fazekas 3

B

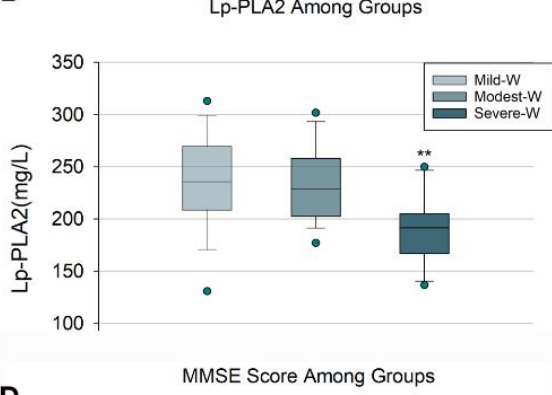

D

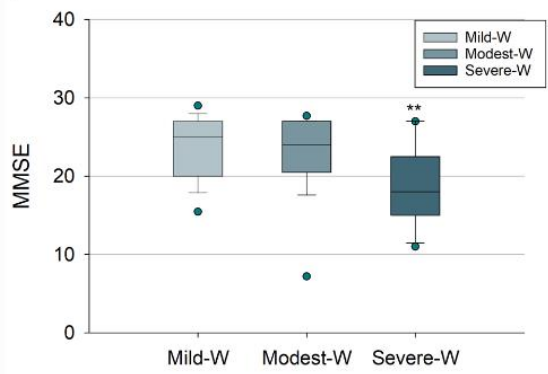

C

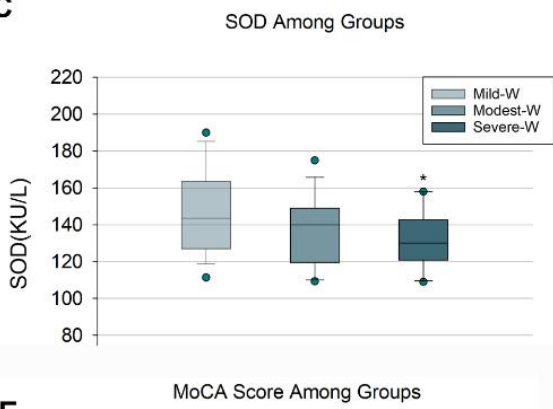

E

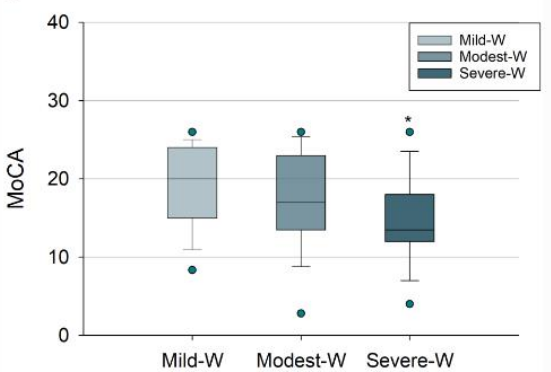

Figure 3. Comparison of Lp-PLA2 and SOD in the CS VD patients with different severity of WMHs. (A) The representative MRI imagings of WMHs in CSVD patients with different severity classified by Fazekas Scores. The levels of Lp-PLA2 (B) and SOD (C) were relatively lower in the CSVD patients of Severe-W compared to those in the Mild-W. The scores of MMSE (D) and MoCA (E) were lower in patients in the Severe-W group compared to those in the MildW group. Abbreviations: CSVD, cerebral small vessel diseases; MMSE, Mini-Mental State Examination; Mild-W, Mild-White matter hyperintensities, MoCA, Montreal Cognitive Assessment; Modest-W, Modest- White matter hyperintensities, MRI, Magnetic Resonance Imaging; Severe-W, Severe-White matter hyperintensities, SOD, Superoxide Dismutase; WMHs, White matter hyperintensities. * Compared to Mild-W $p<0.05$, ** Compared to Mild$\mathrm{W} p<0.01$. 
A

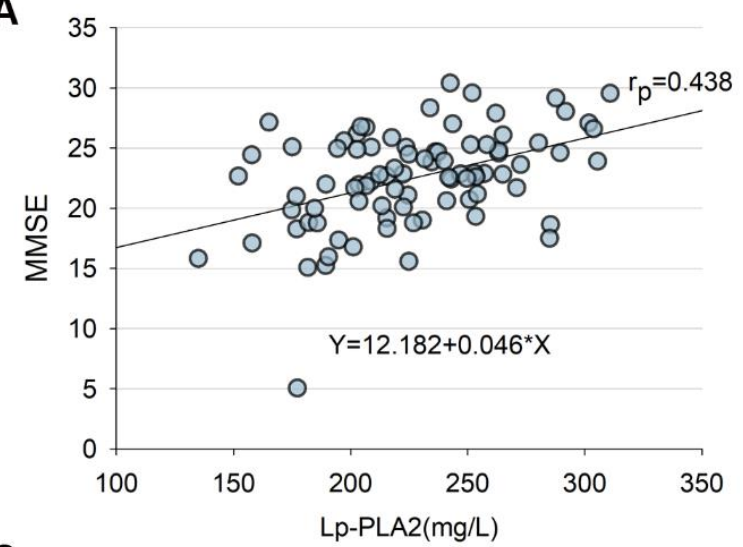

C

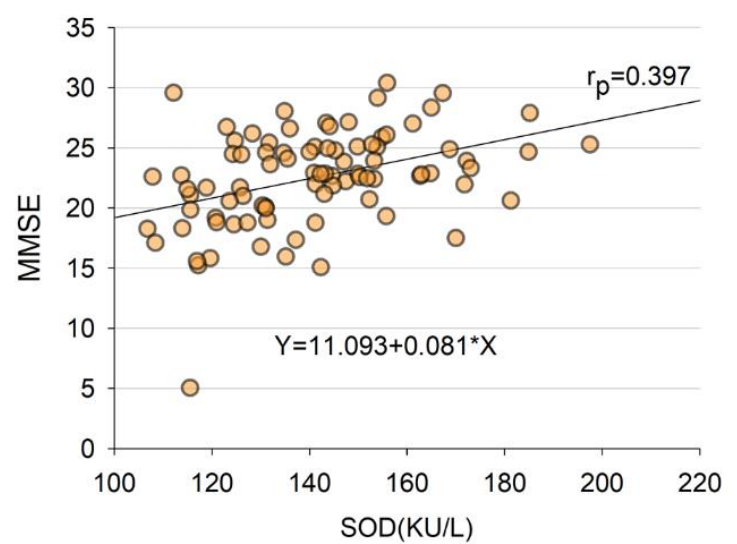

B

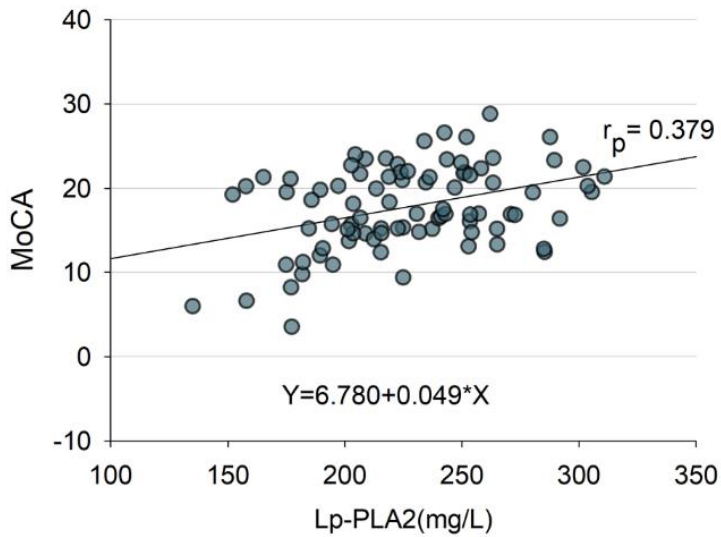

D

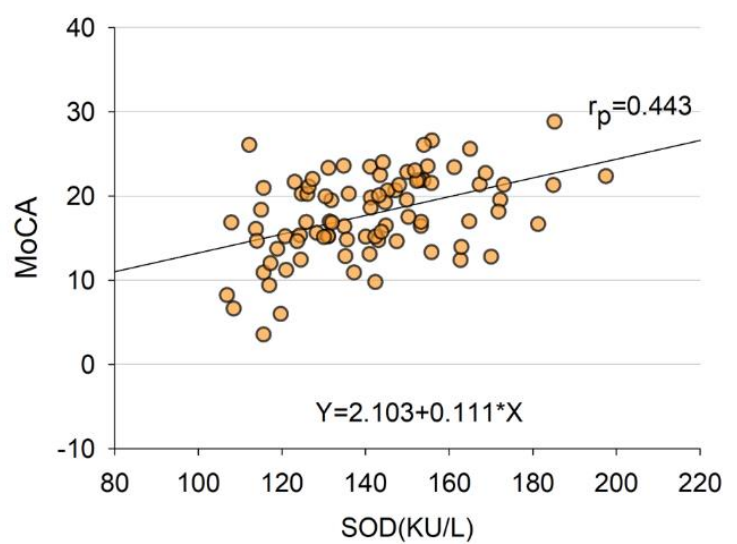

Figure 4. Linear correlation of plasma Lp-PLA2 and SOD with MMSE or MoCA. (A) Lp-PLA2 level in plasma samples were measured. After adjusting for confounders, Lp-PLA2 linear correlated with cognitive impairment severity as measured by MMSE scores $(\mathrm{r}=0.438, p<0.001)$. (B) A similar trend was observed between SOD and MMSE score, and the correlation was statistically significant $(\mathrm{r}=0.379, p<0.01)$. (C) After adjusting for confounders, Lp-PLA2 linear correlated with cognitive impairment severity as measured by MoCA scores $(\mathrm{r}=0.397, p<0.001)$. (D) A similar trend was observed between SOD and MoCA score, and the correlation was statistically signficant $(\mathrm{r}=0.443, p<0.001)$. Abbreviations: Lp-PLA2, Lipoprotein-associated Phospholipase A2; MMSE, Mini-Mental State Examination; MoCA, Montreal Cognitive Assessment; SOD, Superoxide Dismutase.

\section{Lp-PLA2 and SOD as independent factors to predict CI in CSVD}

Ordinal logistic regression and generalized linear models were used to examine the association between the two biomarkers and the extent of cognitive impairment. The results showed that relatively low levels of Lp-PLA2 (OR 0.977, $p=0.003$ ) and SOD (OR 0.933, $p=0.001$ ) were significant independent risk factors for cognitive impairment in CSVD patients as shown in Table 2. The predictive value of other variables, such as education and urea are also shown in Table 2.

ROC analysis of the utility of LP-PLA2 and SOD levels in the diagnosis of CSVD with CI

Due to the interaction between Lp-PLA2 and SOD, the ability of the combination of these two variables to serve as a better diagnostic value was examined. ROC curves were constructed, and the areas under the curves (AUCs) were calculated to assess the value and accuracy of LpPLA2 and SOD for discriminating CSVD patients with MCI from those CSVD patients with NC. The AUCs for Lp-PLA2 and SOD to discriminate between NC and MCI were 0.737 and 0.670 , respectively; however, the combination of Lp-PLA2 and SOD increased the AUC to 0.770. The Youden index was 1.5087 for Lp-PLA2, 1.3783 for SOD, and 1.4957 for their combination (Fig. 5). The value of Lp-PLA2 and SOD for discriminating CSVD patients with SCI from those CSVD patients with NC was also evaluated. The AUCs for Lp-PLA2, SOD, and their combination to discriminate between $\mathrm{NC}$ and SCI were $0.855,0.841 .0 .899$, respectively, while the Youden indices were 1.6483, 1.6739, and 1.7134. The 
sensitivity and specificity for Lp-PLA2 alone was $90.91 \%$ and $73.92 \%$, respectively; however, combined with SOD,

A

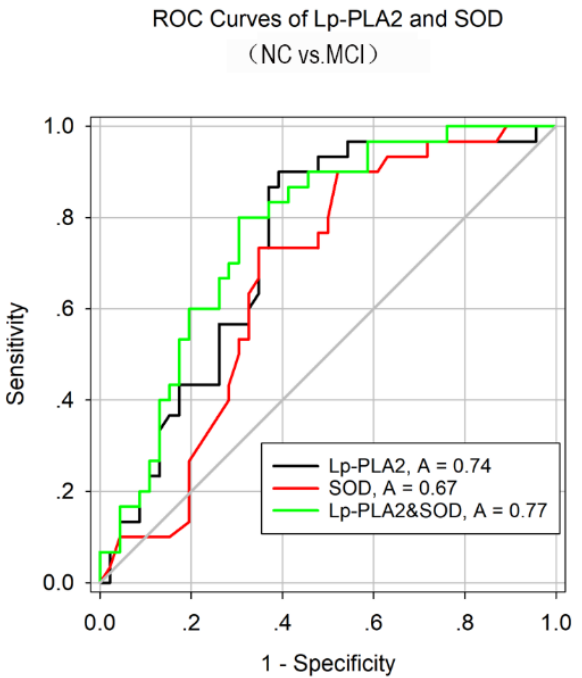

the specificity increased to $80.43 \%$ (Fig. 5).

B ROC Curves of LP-PLA2 and SOD (NC vs.SCl)

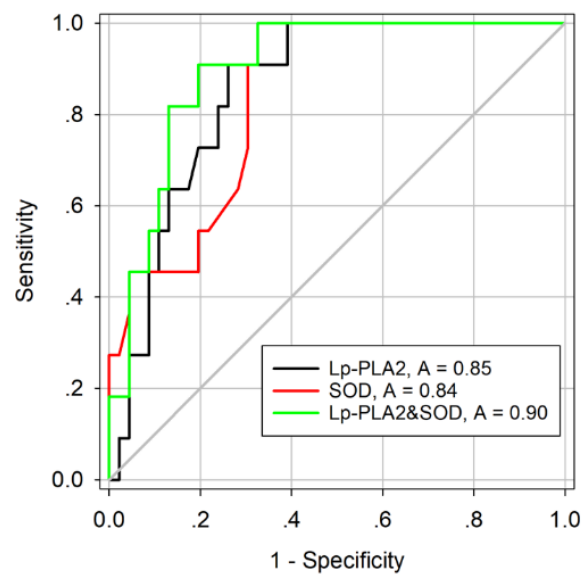

Figure 5. ROC analysis of Lp-PLA2 and SOD. (A) Lp-PLA2 and SOD for CSVD patients with MCI versus CSVD patients with NC. The combined graph showed the Lp-PLA2 (black curve), SOD (red curve) and the combination curve (green curve) respectively. The combination of Lp-PLA2 and SOD was the best discriminating parameters compared to Lp-PLA2 and SOD alone. (B) ROC analy sis of Lp-PLA2 and SOD for CSVD patients with SCI versus NC. The AUC was 0.85 for Lp-PLA2 (black curve), 0.84 for SOD (red curve) and 0.90 for the combination of Lp-PLA2 and SOD (green curve). Abbreviations: CSVD, LpPLA2, Lipoprotein-associated Phospholipase A2; SOD, Superoxide Dismutase; MCI, Mild Cognitive Impairment; SCI, severe cognitive impairment.

\section{DISCUSSION}

Inflammation plays a crucial role in the pathogenesis of cerebral ischemia including CVSD and dementia such as $\mathrm{AD}$ and MCI $[2,20,21]$. Plasma levels of vascular and neuroinflammation-related markers including Lp-PLA2 and SOD have been used as specific measures of cerebrovascular inflammation in ischemic stroke. Although the role of lipoprotein-associated phospholipase A2 in cognitive impairment is known; however, the role of SOD in cognitive impairment especially in CSVD patients remains largely unexplored. In this study, for the first time to our knowledge, we found the relation of LpPLA2 and SOD with cognitive impairment in CSVD patients. Our findings provide several novel observations. First, we note that plasma Lp-PLA2 and SOD levels in CSVD patients with MCI or SCI were lower than those in CSVD patients with $\mathrm{NC}$ and that the two markers were positively correlated to the degree of cognitive impairment in CSVD patients. Second, our study suggested that Lp-PLA2 and SOD may be closely correlated to the severity of white matter lesion in CSVD patients. Third, multiple linear regression showed a significant association between Lp-PLA2/SOD and the degree of cognitive impairment as measured by the
MMSE and MoCA scores in CSVD patients, independent of LDL-C. Fourth, we further confirmed that Lp-PLA2 and SOD were significant independent risk factors for cognitive impairment in CSVD patients as examined by OLRGLMs. Finally, this study created Lp-PLA2 and SOD-related ROC curves to distinguish CSVD patients with MCI from those with NC. Together, these data support the integral roles of the vascular and neuroinflammatory markers Lp-PLA2 and SOD in CSVD patients with cognitive impairment.

Lp-PLA2 is a recognized marker of systemic vascular and neuro inflammation and cerebral/cardiovascular risk, which has been related to ischemic stroke and vascular dementia $[22,23]$. The differences of blood Lp-PLA 2 might be important, as plasma Lp-PLA 2 levels change dynamically early after a cerebral-vascular event and reflect its action in the brain [24]. Several recent studies have examined Lp-PLA2 levels in the acute period after transient ischemic attack (TIA) or stroke and observed elevated levels of plasma Lp-PLA2, which were associated with early recurrent stroke [24, 25]. In this study, excluding other confounders (such as gender, age, years of education, BMI, hypertension, diabetes, CHD, medical statin use, total cholesterol, HDL-C, LDL-C, urea, $\mathrm{Cr}$, UA and APOE), significantly lower levels of Lp- 
PLA2 in CVSD patients with MCI or SCI were observed compared to those in CVSD patients with NC (Fig. 2), and signifcant differences of Lp-LPA2 and SOD were noted among NC, MCI and SCI groups (Table 1). A significant correlation (Fig. 2C) between Lp-PLA2 and SOD was observed, suggesting that there might be a linkage between Lp-PLA2 and SOD in CSVD. These observations suggest that Lp-PLA2 might play important roles in preventing cognitive impairment development in CSVD. This result is consistent with previous findings that indic ated antioxidative effects of Lp-PLA2 [26-28].

Recent findings have indicated that WMHs are assoc iated with the cognitive impairment in CSVD [4, 2931], and our results (Fig. 3D, E) are consistant with these findings. Furthermore, we note that the lower LpPLA2/SOD levels in the severe WMH CSVD patients (Fazekas 3 group) were found compared to those in the mild white matter lesion group (Fazekas 1 group; Fig. 3), stronlgy implying that lower levels of Lp-PLA2/SOD may mediate cognitive impairment in CSVD patients, probably partially via white matter lesion and lacunes. Based on this finding, we ask whether LP-PLA2 and SOD are correlated to cognitive impairment, and whether their lower levels are independently associated factors in mediating cognitive impairment in CSVD patients.

Some prior studies have suggested that the association between Lp-PLA2 and vascular events is strongest in patients with lower LDL-C levels [32] and have found a strong correlation between LDL-C and LpPLA2 in recurrent vascular events [24]. In contrast, another study found no significant difference in the predictive value of Lp-PLA2 based on baseline LDL-C level [33]. In our study, there was evidence of variability in the strong correlation between Lp-PLA2 and cognitive impairment in CSVD patients, which was independent of circulating LDL-C levels (Fig. 4A, B; Table 2), directly indic ating that circulating LDL-C levels in CSVD patients with cognitive impairment may not influence or participate in the disease pathogenesis but rather that circulating Lp-PLA2 may influence CSVD patients via regulating vascular and neuroinflammatory mechanisms but not lipid metabolism.

The relationship between Lp-PLA2 and cognitive impairment reported in other studies has been inconsistent. Savas et al., indicated that Lp-PLA2 levels is higher in healthy subjects compared to $\mathrm{AD}$ patients, implying that Lp-PLA2 may play an anti-inflammatory or antioxidative effects in $\mathrm{AD}$ [34]. A small cross-sectional study of $78 \mathrm{AD}$ cases, 59 amnestic MCI cases, and 66 cognitively normal controls performed by Davidson et al., did not find a significant association between Lp-PLA2 and $\mathrm{AD}$ [35]. One prospective Rotterdam study and Texas Alzheimer's Research and Care Consortium case-control study showed that the levels of Lp-PLA2 were associated with an increased risk of vascular dementia (VD) and $\mathrm{AD}$, independent of other cardiovascular disease (CVD) and inflammatory factors $[23,36]$. The discrepancy between our findings and other studies may be explained by differences in study design, different selected subjectes, data collection, and the number of variables used in the adjusted models to determine in these studies. In the current study, CSVD but not $\mathrm{AD}$ patients were chosen, and we only measured Lp-PLA2 mass. Lp-PLA2 mass detects a portion of the total Lp-PLA2, mainly the LpPLA2 associated with HDL, which is the "good cholesterol" that displays protective effects [16]. In the current study, in the plasma we did not noted any differences of systemically infection-related inflammatory mediators (such as a leukocyte, hs-CRP, neutrophile granulocyte, ESR and uric acid; Table 1) between CSVD patients and healthy subjects. Adjust for those infective and inflammatory potential confounders, and due to the nature and changes in circulating Lp-PLA2 in CSVD patients, our findings suggest that peripheral vascular or neuroinflammatory damage may be a key mediating mechanism by which Lp-PLA2 predisposes CSVD patients to cognitive impairment.

In addition, we observed a similar trend upon examination of the levels of plasma SOD across CSVD groups (Fig. 4C, D), strongly suggesting that lower levels of SOD may be correlated to cognitive impairment, and the lower SOD level is independent risk factors in mediating cognitive impairment in CSVD patients. SOD levels in CSVD patients move in a similar direction as the levels of Lp-PLA2, further confirming that some antiinflammatory and proinflammatory-related markers may behave similarly in certain circumstances. SOD is recognized as an antioxidative stress and antineuroinflammatory-related mediator [37-39]. The gradual downregulation of SOD levels in CSVD patients according to the severity of cognitive impairment and the significant positive correlation between SOD levels and the degree of cognitive impairment strongly suggest that more circulating oxidative stress and inflammatory markers may be present in CSVD subjects with cognitive impairment than in those with NC. These data suggest that plasma SOD levels are not increased in these patients with cognitive impairment; rather, the decline in SOD levels indicates a deficit in antioxidant defense mechanisms in those CSVD subjects with cognitive impairment, since these CSVD patients are unable to remove the circulating superoxide anion, therefore suffering an increase in small vascular damage induced by ROS and neuroinflammation. Thus, a lower level of plasma SOD is associated with increased vascular damage. Due to the retrospective nature of this study, we cannot exclude the possibility that CSVD patients with cognitive impairment produce less SOD and/or that CSVD patients with NC produce more 
SOD. However, longitudinal studies may provide a clue as to whether Lp-PLA2 and SOD play crucial roles in the development of cognitive impairment in subjects with CSVD.

Our results further indicate that only Lp-PLA2 and SOD but not hypertension, UA, Cr, HDL-C, or LDL-C are independent predictors of cognitive impairment in patients with CSVD (Table 2). This interesting finding demonstrates that Lp-PLA2 and SOD may directly participate in the pathogenesis of cognitive impairment in CSVD via regulating vascular and neuroinflammatory damage in circulating blood. Since both Lp-PLA2 and SOD are involved in the neuropathogenesis of CSVD patients and only some but not all CSVD subjects display cognitive impairment, we attempted to determine whether Lp-PLA2 and SOD have enough discriminative power to distinguish CSVD patients with cognitive impairment from those with NC. ROC curve analysis revealed an AUC of 0.737 for Lp-PLA2 and 0.670 for SOD, indicating that both had an acceptable sensitivity and specificity for the potential discrimination of CSVD patients with MCI from those with NC, and Lp-PLA2 had better discrimination accuracy than SOD. The ROC analysis strongly suggests that Lp-PLA2 and SOD can significantly discriminate CSVD patients with MCI from those with $\mathrm{NC}$ and therefore could be used as a valuable diagnostic tool in early screening for cognitive impairment in CSVD subjects. Moreover, the combination of Lp-PLA2 and SOD produced a larger AUC of 0.770 with a sensitivity of $80 \%$ and specificity of $69.7 \%$ in the ROC analysis than Lp-PLA2 or SOD alone, strongly implying that the diagnostic accuracy of the combination of the two variables was superior to that of either variable alone in differentiating CSVD patients with different cognitive status.

The present study is limited mainly by its retrospective nature, assessing plasma levels of Lp-PLA2 and SOD in a relatively small sample size of CSVD patients at a single time point. Prospective and longitudinal studies are needed, and randomized trials of potent reversible pharmacological Lp-PLA2 inhibitors or SOD agonists may help to clarify whether modification of Lp-PLA2 or SOD c an delay the progression of CSVD.

A study strength is that there is very limited evidence that Lp-PLA2 and SOD, especially SOD, are signific ant and independent predictors associated with cognitive impairment in CSVD patients. The observed association of Lp-PLA2 and SOD with CSVD in our study is consistent with the hypothesis that Lp-PLA2 and SOD, as measured in peripheral blood, may reflect vascular and neuroinflammatory mechanisms. The relationship between Lp-PLA2/SOD and CSVD is highly likely to be mediated at least partially via vascular and neuroinflammatory mechanisms. These findings may be important for health care facilities that have limited access to Neurologists specialized in the diagnosis of CSVD with cognitive impairment. Further research in these areas is of great importance because Lp-PLA2 and SOD are modifiable risk factors and, if further confirmed, may be considered as therapeutic targets for preventing cognitive impairment development in CSVD and reducing its severity.

\section{Potential Conflicts of Interest}

The authors declare no conflict of interest.

\section{Acknowledgements}

We greatly thank A/Prof. Longjun Wu and Dr. Dale B. Bosco (Department of Neurology, Mayo Clinic) for their comments on the discussion and critical review of the manuscript. This work was supported by the National Natural Science Foundation of China (Grant NO: 81873777), Science and Technology Program of Guangdong of China (Grant NO: 2016A050502019), Natural Science Foundations of Guangdong of China (Grant NO: 2014A020212068, 2017A030311010), Leading Talent in Talents Project Guangdong High-level Personnel of Special Support Program, and Scientific Research Foundation of Guangzhou (Grant NO: 2014J4100210, 201704030080) to Q.W.; and Natural Science Foundations of Guangdong of China (Grant NO: 2017A030313672) to SZ.

\section{Supplementary Materials}

The Supplemenantry data can be found online at: www.aginganddisease.org/EN/10.14336/AD.2019.0304

\section{References}

[1] Zhao L, Biesbroek JM, Shi L, Liu W, Kuijf HJ, Chu WW, et al. (2018). Strategic infarct location for poststroke cognitive impairment: A multivariate lesionsymptommapping study. J Cereb Blood Flow Metab, 38(8): 1299-1311.

[2] Zhang C, Brandon NR, Koper K, Tang P, Xu Y, Dou H (2018).Invasion of Peripheral Immune Cells into Brain Parenchyma after Cardiac Arrest and Resuscitation. Aging Dis, 9(3): 412-425.

[3] Ter Telgte A, van Leijsen EMC, Wiegertjes K, Klijn CJM, Tuladhar AM, de Leeuw FE (2018). Cerebral small vessel disease: from a focal to a global perspective. Nat Rev Neurol, 14(7): 387-398.

[4] Xu X, Gao Y, Liu R, Qian L, Chen Y, Wang X, et al. (2018).Progression of White Matter Hyperintensities Contributes to Lacunar Infarction. Aging Dis, 9(3): 444-452.

[5] [Hilal S, Mok V, Youn YC, Wong A, Ikram MK, Chen 
CL (2017). Prevalence, risk factors and consequences of cerebral small vessel diseases: data from three Asian countries. J Neurol Neurosurg Psychiatry, 88(8): 669-674.

[6] Walker KA, Power MC, Hoogeveen RC, FolsomAR, Ballantyne CM, Knopman DS, et al. (2017). Midlife Systemic Inflammation, Late-Life White Matter Integrity, and Cerebral Small Vessel Disease: The Atherosclerosis Risk in Communities Study. Stroke, 48(12):3196-3202.

[7] Sternberg Z, Hu Z, Sternberg D, Waseh S, Quinn JF, Wild K, et al. (2017). Serum Hepcidin Levels, Iron Dyshomeostasis and Cognitive Loss in Alzheimer's Dis ease. Aging Dis, 8(2): 215-227.

[8] Swardfager W, Yu D, Ramirez J, Cogo-Moreira H, Szilagyi G, Holmes MF, et al. (2017). Peripheral inflammatory markers indicate microstructural damage within periventricular white matter hyperintensities in Alzheimer's disease: A preliminary report. Alzheimers Dement (Amst), 7:56-60.

[9] Silva IT, Mello AP, and Damasceno NR. (2011). Antioxidant and inflammatory aspects of lipoproteinas s ociated phospholipase A(2)(Lp-PLA(2)): a review. Lipids Health Dis, 10:170.

[10] Gomez-Marcos MA, Blazquez-Medela AM, GamellaPozuelo L, Recio-Rodriguez JI, Garcia-Ortiz L, Martinez-Salgado C (2016). Serum Superoxide Dismutase Is Associated with Vascular Structure and Function in Hypertensive and Diabetic Patients. Oxid Med Cell Longev, 2016:9124676.

[11] Kobylecki CJ, Afzal S, Nordestgaard BG. (2015). Genetically Low Antioxidant Protection and Risk of Cardiovascular Disease and Heart Failure in Diabetic Subjects. EBioMedicine, 2(12): 2010-5.

[12] Melkas S, Putaala J, Oksala NK, Pohjasvaara T, Oksala A, Kaste M, et al. (2011). Small-vess el disease relates to poor poststroke survival in a 12-year followup. Neurology, 76(8):734-9.

[13] Shi L, Zhao L, Yeung FK, Wong SY, Chan RKT, Tse MF, et al. (2018). Mapping the contribution and strategic distribution patterns of neuroimaging features of small vessel dis ease in poststroke cognitive impairment. J Neurol Neurosurg Psychiatry, 89(9): 918-926.

[14] Wardlaw JM, Smith EE, Biessels GJ, Cordonnier C, Fazekas F, Frayne R, et al. (2013). Neuroimaging standards for research into small vessel dis ease and its contribution to ageing and neurodegeneration. Lancet Neurol, 12(8):822-38.

[15] Gorelick PB, Scuteri A, Black SE, Decarli C, Greenberg SM, Iadecola C, et al. (2011). Vascular contributions to cognitive impairment and dementia: a statement for healthcare professionals from the american heart association/american stroke as sociation. Stroke, 42(9): 2672-713.

[16] Zhuo S, Wolfert RL, Yuan C (2017). Biochemical differences in the mass and activity tests of lipoprotein-associated phospholipase A2 explain the dis cordance in results between the two as say methods. Clin Biochem, 50(18): 1209-1215.
[17] Packard CJ, O'Reilly DS, Cas lake MJ, McMahon AD, Ford I, Cooney J, et al. (2000). Lipoprotein-associated phospholipase A2 as an independent predictor of coronary heart disease. West of Scotland Coronary Prevention Study Group. N Engl J Med, 343(16): 1148-55.

[18] Christensen H, BatterhamPJ, Mackinnon AJ, Jorm AF, Mack HA, Mather KA, et al. (2008). The as sociation of APOEgenotype and cognitive decline in interaction with risk factors in a 65-69 year old community sample. BMC Geriatr, 8:14.

[19] Prins ND, Scheltens P (2015). White matter hyperintensities, cognitive impairment and dementia: an update. Nat Rev Neurol, 11(3):157-65. Zhang C, Zhu Y, Wang S, Zachory Wei Z, Jiang MQ, Zhang Y, et al. (2018). Temporal Gene Expression Profiles after Focal Cerebral Is chemia in Mice. Aging Dis, 9(2): 249-261.

[21] King E, O'Brien JT, Donaghy P, Morris C, Barnett N, Olsen K, et al. (2018). Peripheral inflammation in prodromal Alzheimer's and Lewy body dementias. J NeurolNeurosurg Psychiatry, 89(4): 339-345.

[22] Funderburg NT, McComsey GA, Kulkarni M, Bannerman T, Mantini J, Thornton B, et al. (2016). Equivalent Decline in Inflammation Markers with Tenofovir Disoproxil Fumarate vs. Tenofovir Alafenamide. EBioMedicine, 13:321-327.

[23] van Oijen M, van der Meer IM, Hofman A, Witteman JC, Koudstaal PJ, Breteler MM. (2006). Lipoproteinas sociated phospholipase A2 is as sociated with risk of dementia. Ann Neurol, 59(1): 139-44.

[24] Lin J, Zheng H, Cucchiara BL, Li J, Zhao X, Liang X, et al. (2015). Association of Lp-PLA2-A and early recurrence of vascular events after TIA and minor stroke. Neurology, 85(18): 1585-91.

[25] Cucchiara BL, Messe SR, Sansing L, MacKenzie L, Taylor RA, Pacelli J, et al. (2009). Lipoproteinas sociated phospholipase A2 and C-reactive protein for risk-stratification of patients with TIA. Stroke, 40(7): 2332-6.

[26] Noto H, Hara M, Karasawa K, Iso ON, Satoh H, Togo $\mathrm{M}$, et al. (2003). Human plasma platelet-activating factor acetylhydrolase binds to all the murine lipoproteins, conferring protection against oxidative stress. Arterioscler Thromb Vasc Biol, 23(5): 829-35.

[27] Bazan NG. (1995).Inflammation. A signal terminator. Nature, 374(6522): 501-2.

[28] Matsuzawa A, Hattori K, Aoki J, Arai H, Inoue K. (1997). Protection against oxidative stress-induced cell death by intracellular platelet-activating factoracetylhydrolase II. J Biol Chem, 272(51): 32315-20.

[29] Tozer DJ, Zeestraten E, Lawrence AJ, Barrick TR, Markus HS. (2018). Texture Analys is of T1-Weighted and Fluid-Attenuated Inversion Recovery Images Detects Abnormalities That Correlate With Cognitive Decline in Small Vessel Disease. Stroke, 49(7): 16561661.

[30] Yang Y, Kimura-Ohba S, Thompson JF, Salayandia VM, Cosse M, Raz L, et al. (2018). Vascular tight junction disruption and angiogenesis in spontaneously 
hypertensive rat with neuroinflammatory white matter injury. NeurobiolDis, 114: 95-110.

[31] Lampe L, Kharabian-Masouleh S, Kynast J, Arelin K, Steele CJ, Loffler M, et al. (2019). Lesion location matters: The relationships between white matter hyperintensities on cognition in the healthy elderly. $\mathrm{J}$ Cereb Blood Flow Metab, 39(1): 36-43.

[32] Elkind MS, Tai W, Coates K, Paik MC, Sacco RL. (2006). High-sensitivity C-reactive protein, lipoprotein-associated phospholipase A2, and outcome after ischemic stroke. Arch Intern Med, 166(19): 2073-80.

[33] Oei HH, van der Meer IM, Hofman A, Koudstaal PJ, Stijnen T, Breteler MM, et al. (2005). Lipoproteinassociated phospholipase A2 activity is associated with risk of coronary heart disease and is chemic stroke: the Rotterdam Study. Circulation, 111(5): 570-5.

[34] Savas S, Kabaroglu C, Alpman A, Sarac F, Yalcin MA, Parildar Z, et al. (2016). No relationship between lipoprotein-associated phospholipase A2, proinflammatory cytokines, and neopterin in Alzheimer's disease. Exp Geronto1, 77:1-6.

[35] Davidson JE, Lockhart A, Amos L, Stirnadel-Farrant HA, Mooser V, Sollberger M, et al. (2012). Plasma lipoprotein-associated phospholipase A2 activity in Alzheimer's disease, amnestic mild cognitive impairment, and cognitively healthy elderly subjects: a cross-sectional study. Alzheimers Res Ther, 4(6): 51.

[36] Doody RS, Demirovic J, Ballantyne CM, Chan W, Barber R, Powell S, et al. (2015). Lipoproteinassociated phospholipase A2, homocysteine, and Alzheimer's disease. Alzheimers Dement (Amst), 1(4): 464-71.

[37] Shvil N, Banerjee V, Zoltsman G, Shani T, Kahn J, Abu-Hamad S, et al. (2018). MIF inhibits the formation and toxicity of misfolded SOD1 amyloid aggregates: implications for familial ALS. Cell Death Dis, 9(2): 107.

[38] Xu X, Zhang B, Lu K, Deng J, Zhao F, Zhao BQ, et al. (2016). Prevention of Hippocampal Neuronal Damage and Cognitive Function Deficits in Vascular Dementia by Dextromethorphan. Mol Neurobiol, 53(5): 34943502 .

[39] Al-Owais MM, Hettiarachchi NT, Boyle JP, Scragg JL, Elies J, Dallas ML, et al. (2017). Multiple mechanisms mediating carbon monoxide inhibition of the voltagegated $\mathrm{K}(+)$ channel Kv1.5. Cell Death Dis, 8(11): e3163. 